Op den Kamp, J. A. F., de Gier, J., \& van Deenen, L. L. M. (1974) Biochim. Biophys. Acta 345, 253-256.

Op den Kamp, J. A. F., Kauerz, M. Th., \& van Deenen, L. L. M. (1975) Biochim. Biophys. Acta 406, 169-177.

Rance, M., \& Byrd, R. A. (1983) J. Magn. Reson. 52, 221-240.

Rand, R. P., Das, S., \& Parsegian, V. A. (1985) Chem. Scr. $25,15-21$.

Rink, T. J., Sanchez, A., \& Hallam, T. J. (1983) Nature 305 , 317-319.

Salmon, A., Dodd, S. W., Williams, G. D., Beach, J. M., \& Brown, M. F. (1987) J. Am. Chem. Soc. 109, 2600-2609.

Seelig, A., \& Seelig, J. (1974) Biochemistry 13, 4839-4845.

Seelig, J. (1977) Q. Rev. Biophys. 10, 353-418.

Seelig, J. (1978) Biochim. Biophys. Acta 515, 105-140.
Stockton, G. W., Polnaszek, C. F., Tulloch, A. P., Hasan, F., \& Smith, I. C. P. (1976) Biochemistry 15, 954-966.

Takuwa, N., Takuwa, Y., \& Rasmussen, H. (1987) Biochem. J. 243, 647-653.

Thayer, A. M., \& Kohler, S. J. (1981) Biochemistry 20, 6831-6834.

Wakelam, M. J. O. (1985) Biochem. J. 228, 1-12.

Wassall, S. R., Thewalt, J. L., Wong, L., Gorrissen, H., \& Cushley, R. J. (1986) Biochemistry 25, 319-326.

Whitaker, M., \& Aitchison, M. (1985) FEBS Lett. 182, 119-124.

Wilschut, J. C., Regts, J., Westenberg, H., \& Scherphof, G. (1978) Biochim. Biophys. Acta 508, 185-196.

Zidovetzki, R., Banerjee, U., Harrington, D. W., \& Chan, S. I. (1988) Biochemistry 27, 5686-5692.

\title{
Spin-Label ESR Studies of Lipid-Protein Interactions in Thylakoid Membranes
}

\author{
Gang Li, ${ }^{\ddagger}$ Peter F. Knowles, ${ }^{*, \ddagger}$ Denis J. Murphy, ${ }^{\S}$ Ikuo Nishida," and Derek Marsh ${ }^{\perp}$ \\ Astbury Department of Biophysics, University of Leeds, Leeds LS2 9JT, U.K., Department of Biological Sciences, Durham \\ University, Durham DHI 3LE, U.K., National Institute for Basic Biology, Okazaki, 444 Japan, and Max-Planck-Institut für \\ biophysikalische Chemie, Abteilung Spektroskopie, D-3400 Göttingen, FRG \\ Received January 25, 1989; Revised Manuscript Received May 19, 1989
}

\begin{abstract}
Lipid-protein interactions in thylakoid membranes, and in the subthylakoid membrane fractions containing either photosystem 1 or photosystem 2 , have been studied by using spin-labeled analogues of the thylakoid membrane lipid components, monogalactosyldiacylglycerol, phosphatidylglycerol, and phosphatidylcholine. The electron spin resonance spectra of the spin-labeled lipids all consist of two components, one corresponding to the fluid lipid environment in the membranes and the other to the motionally restricted membrane lipids interacting directly with the integral membrane proteins. Spectral subtraction has been used to quantitate the fraction of the membrane lipids in contact with the membrane proteins and to determine the selectivity between the different lipid classes for the lipid-protein interaction. The fractions of motionally restricted lipid in the thylakoid membrane are $0.36,0.39$, and 0.53 , for the spin-labeled monogalactosyldiacylglycerol, phosphatidylcholine, and phosphatidylglycerol, respectively. Spin-labeled monogalactosyldiacylglycerol exhibits very little preferential interaction over phosphatidylcholine, which suggests that part of the role of monogalactosyldiacylglycerol in thylakoid membranes is structural, as is the case for phosphatidylcholine in mammalian membranes. Spin-labeled phosphatidylglycerol shows a preferential interaction over the corresponding monogalactosyldiacylglycerol and phosphatidylcholine analogues, in contrast to the common behavior of this lipid in mammalian systems. This pattern of lipid selectivity is preserved in both the photosystem 1 and photosystem 2 enriched subthylakoid membrane fractions.
\end{abstract}

$\mathbf{T}_{\text {h }}$ series of integral protein complexes embedded in the thylakoid membrane. The conversion of light energy into a chemically useful form takes place at two reaction centers, photosystem 1 (PS1) $^{1}$ and photosystem 2 (PS2), which consist of complexes of different integral proteins. In the thylakoids from the mesophyll cells of higher plants, the two reaction centers and associated protein complexes are separately located in the appressed (PS2) and nonappressed (PS1) membrane regions. The appressed membranes are arranged in stacks and are interconnected by the nonappressed membranes, which contain regions of high membrane curvature. Within the thylakoid,

\footnotetext{
$\ddagger$ University of Leeds.

Durham University.

N National Institute for Basic Biology.

$\perp$ Max-Planck-Institut.
}

the membrane lipids presumably have an important role to play in stabilizing this structural arrangement and, via the lipid-protein interactions, in integrating the protein complexes and possibly in maintaining their spatial distribution. The very active metabolic state of thylakoid membranes is reflected by their relatively high protein content $[65-75 \%(w / w)$ protein and pigments], the remainder being acyl lipid.

In addition to the specialized protein composition, the thylakoid membrane has a characteristic lipid composition that

\footnotetext{
I Abbreviations: 12-MGDGSL, 1-oleoyl-2-[12-(4,4-dimethyloxazolidine- $N$-oxyl)stearoyl]-sn-glycero-3-galactose; 14-PGSL, 1 -acyl2-[14-(4,4-dimethyloxazolidine- $N$-oxyl)stearoyl]-sn-glycero-3-phosphoglycerol; $n$-PCSL, 1 -acyl-2-[ $n$-(4,4-dimethyloxazolidine- $N$-oxyl)stearoyl]-sn-glycero-3-phosphocholine; PS1, photosystem 1; PS2, photosystem 2; EDTA, ethylenediaminetetraacetic acid; HEPES, $N$-(2hydroxyethyl)piperazine- $N^{\prime}$-2-ethanesulfonic acid; MES, 2- $(N$ morpholino)ethanesulfonic acid; ESR, electron spin resonance.
} 
differs radically from that of biological membranes of nonplant origin. Instead of phospholipids, the major lipids in the thylakoid membrane are glycolipids, which account for approximately $80 \%$ of the total acyl lipids. Typically, the galactolipids (mono- and digalactosyldiacylglycerol) account for $68 \%$ of the acyl lipids, negatively charged lipids (sulfoquinovosyldiacylglycerol and phosphatidylglycerol) make up $24 \%$ of the acyl lipids, and phospholipids (phosphatidylglycerol and phosphatidylcholine) account for approximately $19 \%$ of the total acyl lipids (Gounaris et al., 1983; Nishihara et al., 1980; Chapman et al., 1983). Of the galactolipids, monogalactosyldiacylglycerol is particularly interesting, since when dispersed alone in water it does not form bilayer membranes, but inverted hexagonal phases (Gounaris \& Barber, 1983). This property of the MGDG lipid component may be necessary for creating the unique structure of the thylakoid membrane stacks, as well as for integrating the photosynthetic reaction center proteins into the membrane. Of the other lipids, the negatively charged lipids may also have a special role, since it has been found in many other membrane systems that most anionic lipids display a specificity for the interaction with integral membrane proteins [see, e.g., Marsh (1987)]

Although the thylakoid membrane is one of the most abundant membranes in the biosphere [cf. Gounaris et al. (1986)], relatively few studies have been undertaken that relate to the lipid-protein interactions that are essential for the membrane integrity. This is especially seen to be the case when comparison is made with the situation for membranes of nonplant origin. Spin-label ESR spectroscopy has been found to be particularly useful in the investigation of lipid-protein interactions in other membrane systems. Both the stoichiometry and selectivity of interaction of spin-labeled lipids with integral proteins, as well as the effect of the lipid-protein interaction of the lipid chain mobility, are amenable to detailed study by this technique [see, e.g., Marsh (1983, 1985)].

In the present work, we have investigated the lipid-protein interactions both in intact thylakoid membranes and in subthylakoid membrane fractions enriched in either photosystem 1 or photosystem 2. ESR spectroscopy in combination with spin-labeled analogues of the lipids present in thylakoid membranes, in particular monogalactosyldiacylglycerol, has been used for this study. This has allowed the specificity of the lipid-protein interactions to be analyzed and the effects of the lipid-protein interaction on the lipid mobility to be characterized. A marked lipid specificity has been observed for phosphatidylglycerol, which might extend to the other negatively charged lipids of the thylakoid membrane. The lipid specificity pattern is found to be similar in the membrane fractions enriched in either PS1 or PS2 centers, but quantitative differences in the degree of lipid association between the two centers are observed, corresponding to the different protein and lipid compositions of the appressed and nonappressed regions of the thylakoid membrane.

\section{Materials AND Methods}

Spin-labeled phosphatidylcholines ( $n$-PCSL) were prepared from the corresponding spin-labeled stearic acids and egg lysophosphatidylcholine as described in Marsh and Watts (1982). Spin-labeled phosphatidylglycerol (14-PGSL) was prepared from the corresponding spin-labeled phosphatidylcholine by transphosphatidylation catalyzed with phospholipase $\mathrm{D}$, as described in the same reference. Spin-labeled monogalactosyldiacylglycerol (12-MGDGSL) was prepared as described by Nishida and Yamada (1985). This synthesis yields $30 \%$ chain migration, which is considerably greater than that for the phospholipid labels.
Pea seeds (Pisum sativum L., var. Kelvedon Wonder) were directly germinated in vermiculite, kept in the dark for the first 5 days at $25^{\circ} \mathrm{C}$, and then transferred to a controlled growth room $\left(20^{\circ} \mathrm{C}, 12 \mathrm{~h}\right.$ light/dark). Leaves were harvested after approximately 14 days of growth, following a $12 \mathrm{~h}$ dark/1 $\mathrm{h}$ light period to reduce starch levels. The leaves were homogenized in grinding medium $(330 \mathrm{mM}$ glucose, $50 \mathrm{mM}$ $\mathrm{Na}_{2} \mathrm{HPO}_{4}, 50 \mathrm{mM} \mathrm{KH} \mathrm{PO}_{4}, 5 \mathrm{mM} \mathrm{MgCl}, 25 \mathrm{mM} \mathrm{NaCl}, \mathrm{pH}$ $6.5)$ with two bursts of $2 \mathrm{~s}$ in a chilled blender. The homogenate was filtered through eight layers of muslin and the filtrate centrifuged briefly $(30 \mathrm{~s}, 2500 \mathrm{~g})$ to pellet the chloroplasts. The pellets were resuspended in a small volume of 5 $\mathrm{mM} \mathrm{MgCl} 2$ and vortexed for $20-60 \mathrm{~s}$. The stacked thylakoids were then twice washed free of lysed chloroplast envelopes by further centrifugation, after resuspension in $330 \mathrm{mM}$ sorbitol, $2 \mathrm{mM}$ EDTA, $5 \mathrm{mM} \mathrm{MgCl}$, $1 \mathrm{mM} \mathrm{MnCl}$, and $50 \mathrm{mM}$ HEPES, pH 7.6. The final pellets were resuspended in the same buffer and kept in the dark at $4^{\circ} \mathrm{C}$ until use (usually within $30 \mathrm{~min}$ ).

The chlorophyll $a / b$ ratio of both leaves and thylakoid preparations was measured by the method of Arnon (1949). Values for the thylakoid preparations were similar to those for the intact leaves (ca. 2.4). Any preparations with appreciably lower values were discarded. The protein content of the thylakoid preparation was measured by the method of Markwell et al. (1981), and the lipid content was determined by gas chromatographic and gravimetric analysis as described in Murphy et al. (1988). The lipid/chlorophyll/protein ratio was determined to be $33 / 8 / 58(\mathrm{w} / \mathrm{w} / \mathrm{w})$. The intactness and purity of the thylakoid preparations were also checked by thin-section electron microscopy, according to the methods of Telfer et al. (1976), except that the resin used for embedding was obtained from TAAB Laboratory Equipment Services. The electron micrographs showed that the preparations were essentially free of contamination by other membranes and organelles.

Total lipids and chlorophyll were extracted from thylakoids by a modification of the method of Waggoner et al. (1985). One volume of thylakoid suspension ( $3 \mathrm{mg}$ of chlorophyll $/ \mathrm{mL}$ ) in distilled water was mixed by vortexing with 20 volumes of $2: 1(\mathrm{v} / \mathrm{v})$ chloroform/methanol. Six volumes of $0.7 \% \mathrm{NaCl}$ aqueous solution was added with vortexing, and the lower chloroform phase was separated and filtered. Polar lipids were separated from chlorophylls and other nonpolar lipids by chromatography on silica gel $\mathrm{G}$. The column was packed with hexane/chloroform/2-propanol 30:65:5 (v/v/v). The thylakoid total lipid extract was loaded in a small volume of the packing solvent mixture and sequentially eluted with hexane/chloroform/2-propanol 30:65:5, hexane/chloroform/2-propanol $5: 65: 15$, chloroform/2-propanol/methanol 75:15:1, and finally chloroform/methanol 5:1. Eluate fractions were monitored by thin-layer chromatography. All fractions eluting after the pigments and other nonpolar lipids were combined as polar lipids, which correspond to monogalactosyldiacylglycerol and all other thylakoid lipids of higher polarity.

Photosystem 1 enriched membranes were prepared from isolated thylakoids by Yeda press treatment essentially according to the method of Sane et al. (1970). Thylakoid membranes were resuspended in stacking medium $(330 \mathrm{mM}$ sorbitol, $5 \mathrm{mM} \mathrm{MgCl} 2,15 \mathrm{mM} \mathrm{NaCl}, 50 \mathrm{mM}$ HEPES, $\mathrm{pH}$ 7.0) and incubated in the dark at $4^{\circ} \mathrm{C}$ for $90 \mathrm{~min}$. The suspension was then diluted to a concentration of $1 \mathrm{mg}$ of chlorophyll $/ \mathrm{mL}$ and passed dropwise three times through a Yeda press at a pressure of approximately $11 \mathrm{MN} / \mathrm{m}^{2}$. The suspension of ruptured membranes was centrifuged (45 min, 
$40000 \mathrm{~g}$ ) to remove thylakoids and granal membranes. The supernatant was centrifuged at $100000 \mathrm{~g}$ for $1 \mathrm{~h}$, and the resulting PS1-enriched membrane pellets were resuspended in $300 \mathrm{mM}$ sucrose, $5 \mathrm{mM} \mathrm{MgCl}, 15 \mathrm{mM} \mathrm{NaCl}$, and $20 \mathrm{mM}$ HEPES, $\mathrm{pH} 7.0$. The lipid/chlorophyll/protein ratio of the PS1-enriched membranes was found to be 33:4:63 (w/w/w).

Photosystem 2 enriched membranes were prepared by detergent extraction according to the method of Berthold et al. (1981). A suspension of stacked thylakoids prepared as above was diluted to a concentration of $2 \mathrm{mg}$ of chlorophyll $/ \mathrm{mL}$ and incubated in the dark for $25 \mathrm{~min}$ after addition of Triton X-100 at a concentration of $50 \mathrm{mg} / \mathrm{mL}$. The PS2-enriched membranes were then harvested by centrifugation at $40000 \mathrm{~g}$ for $30 \mathrm{~min}$ and the membrane pellets resuspended in the same buffer as used for the PS1-enriched membranes. The lipid/chlorophyll/protein ratio of the PS2-enriched membranes was determined to be $16: 17: 67(\mathrm{w} / \mathrm{w} / \mathrm{w})$.

The purity of the subthylakoid membrane preparations was checked by polyacrylamide gel electrophoresis. The gel patterns of the PS1-enriched preparations contained very little of the $24-28-\mathrm{kDa}$ polypeptides characteristic of the PS2LHC2 centers, whereas the PS2-enriched preparations contained very little of the $50-55-\mathrm{kDa}$ polypeptides characteristic of the PS1 reaction centers. Thus, there was little crosscontamination of the two photosystem complexes (Murphy, 1986).

For spin-labeling, thylakoid membranes or subthylakoid fragments comprising approximately 1-2 $\mathrm{mg}$ of polar lipids were suspended in 2-3 $\mathrm{mL}$ of $20 \mathrm{mM}$ MES, $50 \mathrm{mM} \mathrm{KCl}$, and $5 \mathrm{mM} \mathrm{MgCl}_{2}, \mathrm{pH} 6.5$, and $20 \mu \mathrm{L}$ of $1 \mathrm{mg} / \mathrm{mL}$ spin-label solution in ethanol was added slowly. The sample was then vortexed briefly and incubated for 15-20 min in the dark at room temperature. The spin-labeled sample was then centrifuged $\left(45 \mathrm{~min}, 90000 \mathrm{~g}, 4^{\circ} \mathrm{C}\right.$ ) and washed in $13 \mathrm{~mL}$ of the same buffer by using a Beckmann SW-40 swing-out rotor. Where necessary, the sample was rewashed with the buffer required for the ESR experiment. The pellet from the final centrifugation was transferred with about $40 \mu \mathrm{L}$ of buffer to a $100-\mu \mathrm{L}$ capillary sealed at one end. The sample was then packed by using a bench-top centrifuge $(2000 \mathrm{~g}$ for $5 \mathrm{~min}$ ), excess buffer removed, and the capillary completely sealed. The capillary tubes were wrapped with aluminum foil throughout these operations to minimize the light-induced ESR signal [an asymmetric singlet, close to the central line of the nitroxide spectrum (Warden, 1978)]. To minimize spin-label reduction with PS1-containing preparations, spin-labeling, sample preparation, and ESR measurements were performed either in the dark or under dim green light.

The polar lipid extract ( $3 \mathrm{mg}$ ) was spin labeled by codissolving with $0.015 \mathrm{mg}$ of spin label in chloroform/methanol $1: 1(\mathrm{v} / \mathrm{v})$. The solvents were removed under nitrogen flow and dried under vacuum for at least $3 \mathrm{~h}$. The dry lipid was then hydrated with $50 \mu \mathrm{L}$ of MES buffer by warming to $40^{\circ} \mathrm{C}$, vortexing, and sonicating in a bath sonicator, under nitrogen in a capped tube.

For ESR measurements, the 1-mm-i.d. sample capillaries were centered in a standard 4-mm quartz ESR tube that contained light silicon oil for thermal stability. ESR spectra were recorded by using a Bruker ER200 9-GHz spectrometer with rectangular TE102 cavity and equipped with nitrogen gas flow temperature control to $\pm 0.5^{\circ} \mathrm{C}$. The spectrometer was interfaced to an Acorn 1770 DFS microcomputer with Microlink interface (Biodata, Manchester, U.K.) via an IEEE-488 bus. Spectral subtraction and addition were performed essentially as described in Marsh (1982). Pairwise intersub-

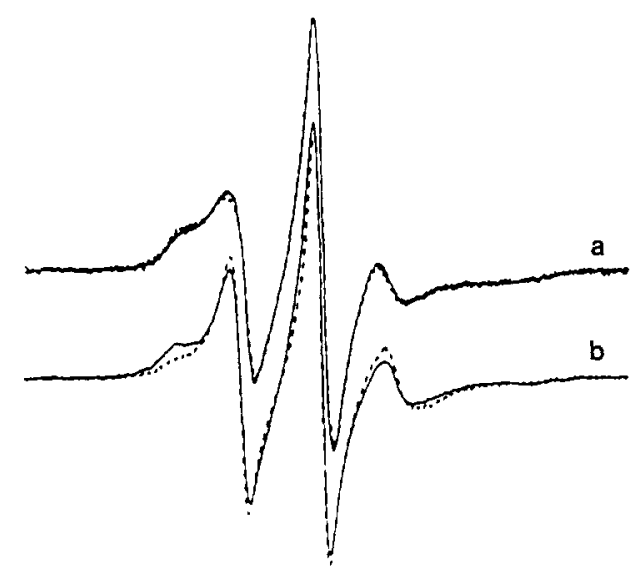

FIGURE 1: ESR spectra of lipid spin labels in thylakoid membranes at $18^{\circ} \mathrm{C}$. (a) 12-MGDGSL monogalactosyl diacylglycerol spin label (full line) and 12-PCSL spin label (dashed line). (b) 14-PGSL phosphatidylglycerol spin label (full line) and 14-PCSL phosphatidylcholine spin label (dashed line). Total scan width was $100 \mathrm{G}$.

tractions were analyzed as described in Knowles et al. (1981). Relative spin-label intensities in the different spectral components were determined by double integration of the firstderivative spectra after base-line correction.

\section{RESULTS}

The water-splitting enzyme in photosystem 2 contains a $\mathrm{Mn}^{2+}$ ion that gives rise to an ESR spectrum at room temperature that is centered around $g=2$. This background signal (which was small in fresh thylakoids but more intense in the PS2-enriched membranes) was removed from the lipid spin label ESR spectra of thylakoid and photosystem 2 enriched membranes by spectral subtraction using unlabeled membrane preparations. Any light-induced ESR signals from the photosystems were similarly removed. Comparison of the ESR spectra of thylakoid preparations that had been frozen in liquid nitrogen for $24 \mathrm{~h}$ with those from fresh preparations revealed an additional spin-spin broadened component on spin-labeling the former samples. All spectra reported here were derived from fresh thylakoid preparations.

ESR spectra of the monogalactosyldiacylglycerol, phosphatidylglycerol, and phosphatidylcholine spin labels in thylakoid membranes at $18^{\circ} \mathrm{C}$ are given in Figure 1. As in many other lipid-protein systems studied [see, e.g., Marsh (1983, 1985)], the spectra for all labels consist of two components corresponding to lipid environments of different mobilities. One component, in the central region of the spectrum, corresponds to spin labels in a fluid lipid environment. The spectral line shape is similar to that obtained from the same labels in an aqueous dispersion of the extracted membrane lipids, as can be seen from the spectra of the 14-positional isomers in the total polar lipid extract in Figure 2. The second component, which is visible in the outer wings of the spectrum, corresponds to lipids whose motion is restricted by interaction with the integral membrane proteins. Various studies on the lipid/protein ratio dependence in reconstituted systems have indicated that this component bears a fixed stoichiometry to the amount of protein present and therefore is attributed to lipids in direct contact with the intramembranous surface of the protein [see, e.g., Marsh $(1983,1985)]$. The two-component nature of the spectra can be further substantiated by spectral subtraction, as demonstrated later.

Comparison between the spectra of the various spin-labeled lipids in the thylakoid membranes in Figure 1 shows that the relative proportions of the motionally restricted and fluid lipid components vary between the different lipid species. These 


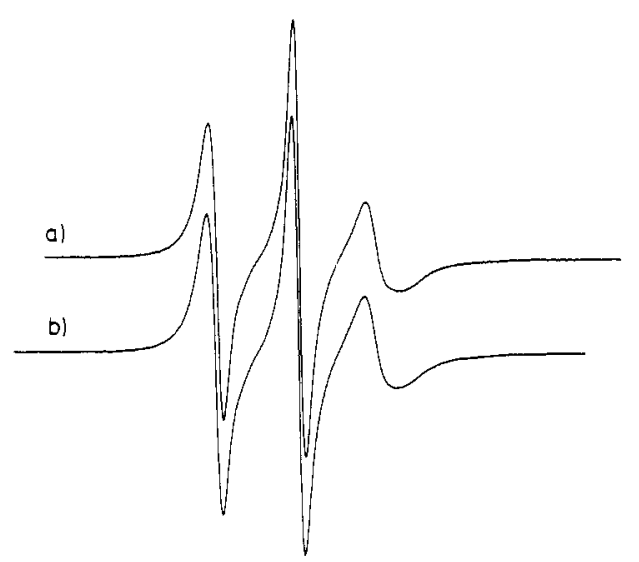

FIGURE 2: ESR spectra of lipid spin labels in aqueous dispersions of the total polar lipid extract from thylakoid membranes. (a) 14-PGSL spin label. (b) 14-PCSL spin label. $T=18^{\circ} \mathrm{C}$; total scan width was $100 \mathrm{G}$.

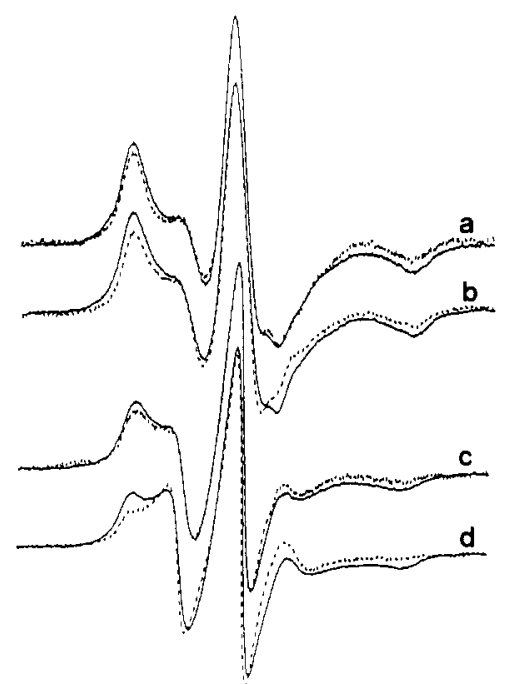

FIGURE 3: ESR spectra of lipid spin labels in PS2-eniched membranes produced by detergent extraction. (a) 12-MGDGSL spin label (full line) and 12-PCSL spin label (dashed line) at $18^{\circ} \mathrm{C}$. (b) 14-PGSL spin label (full line) and 14-PCSL spin label (dashed line) at $18^{\circ} \mathrm{C}$ (c) 12-MGDGSL spin label (full line) and 12-PCSL spin label (dashed line) at $39^{\circ} \mathrm{C}$. (d) 14-PGSL spin label (full line) and 14-PCSL spin label (dashed line) at $39^{\circ} \mathrm{C}$. Total scan width was $100 \mathrm{G}$.

differences correspond to selectivities in the lipid-protein interactions between the different lipid headgroup types. Slightly less 12-MGDGSL than 12-PCSL is associated with the protein, and considerably more 14-PGSL than 14-PCSL is motionally restricted by the protein.

ESR spectra of the monogalactosyldiacylglycerol, phosphatidylglycerol, and phosphatidylcholine spin labels in PS2enriched membranes produced by detergent extraction are given in Figure 3. Since the detergent treatment yields membranes with a reduced lipid content, the spectra are dominated in all cases by the motionally restricted lipid component. Only for the 14-PCSL label at $39^{\circ} \mathrm{C}$ can both spectral components be clearly distinguished (Figure $3 d$ ). In each case, however, the increase in intensity in the central region of the $39^{\circ} \mathrm{C}$ spectrum, relative to the $18^{\circ} \mathrm{C}$ spectrum, indicates the presence of a fluid component. Quantitation was performed by spectral subtraction with the spectra recorded at $39^{\circ} \mathrm{C}$, which allowed resolution of a clearly defined fluid component as a subtraction end point in all cases. Phosphatidylglycerol, but not monogalactosyldiacylglycerol, displays a preference relative to phosphatidylcholine for the protein, as was the case with the whole thylakoids.

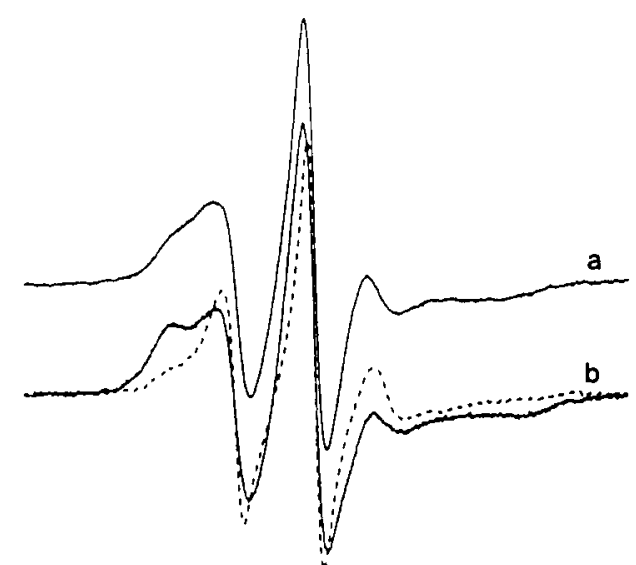

FIGURE 4: ESR spectra of lipid spin labels in PS1-enriched membranes produced by Yeda press treatment, recorded at $12^{\circ} \mathrm{C}$. (a) $12-$ MGDGSL spin label. (b) 14-PGSL spin label (full line) and 14-PCSL spin label (dashed line). Total scan width was $100 \mathrm{G}$.

Experiments with PS2-enriched membranes produced by Yeda press treatment yield a higher proportion of the fluid lipid component for 14-PGSL than do the membranes produced by detergent extraction (data not shown). This confirms that the higher proportion of the motionally restricted component in Figure 3 results from the lower lipid/protein ratio of the detergent-extracted membranes.

ESR spectra of the monogalactosyldiacylglycerol, phosphatidylglycerol, and phosphatidylcholine spin labels in PS1enriched membranes produced by Yeda press treatment are given in Figure 4. The spectra here all consist of two components corresponding to the two different lipid environments. A clear selectivity of phosphatidylglycerol relative to phosphatidylcholine is evident also in these spectra (Figure 4b). The fraction of motionally restricted lipid for the 12 MGDGSL spin label is comparable to that in thylakoids (cf. Figure 1a).

The relative proportions of the motionally restricted and fluid components in the ESR spectra of the lipid/protein systems have been quantitated both by spectral subtraction and by addition of the individual components [see, e.g., Marsh $(1983,1985)]$. Additionally, pairwise intersubtraction between lipid/protein samples with different proportions of the two components has been used. Some difficulty was experienced in obtaining appropriate spectra for the individual components. The spectra of the isolated polar membrane lipids (Figure 2), for example, were found not to be suitable for the fluid component, presumably because of the effects of slow exchange and longer range perturbation of the lipid in the proteincontaining samples. For this reason, the single-component spectra were determined by pairwise intersubtractions between thylakoid and PS2 spectra. The validity of the single-component end points was demonstrated by the agreement between independent intersubtractions using different pairs of spectra.

Representative single-component subtractions and additions for the spectrum of 12-MGDGSL in thylakoid membranes are given in Figure 5. The complementary methods of subtraction (using either the fluid or motionally restricted component) and spectral addition give consistent results and indicate that $36 \%$ of the total spin-label intensity is in the motionally restricted component. The values of the fraction of motionally restricted lipid, $f$, for the different spin labels in thylakoid membranes, obtained by the different methods of spectral analysis, are given in Table I. As in Figure 5, these various methods yielded rather similar values in all cases. In addition, the independent values derived from the 12-PCSL and 14-PCSL positional 
Table I: Fraction of Motionally Restricted Spin-Labeled Lipid Components, $f$, in Thylakoid Membranes Determined by the Different Methods of ESR Spectral Analysis

\begin{tabular}{lcccc}
\hline & \multicolumn{4}{c}{ spin label } \\
\cline { 2 - 5 } \multicolumn{1}{c}{ method } & $\begin{array}{c}12- \\
\text { MGDGSL }\end{array}$ & PCSL & PCSL & PGSL \\
\hline addition & 0.36 & 0.42 & 0.38 & 0.54 \\
fluid end point & 0.36 & 0.39 & 0.40 & 0.54 \\
restricted end point & 0.36 & 0.40 & 0.37 & 0.54 \\
intersubtraction & 0.34 & 0.41 & 0.34 & 0.50 \\
\hline
\end{tabular}

Table II: Fraction of Motionally Restricted Spin-Labeled Lipid Components, $f$, in Thylakoid, PS2-Enriched, and PS1-Enriched Membranes Determined by ESR Spectral Analysis ${ }^{a}$

\begin{tabular}{cccc}
\hline membrane & 12-MGDGSL & PCSL $^{b}$ & 14-PGSL \\
\hline thylakoid & 0.36 & 0.39 & 0.53 \\
PS2-enriched & 0.63 & 0.63 & 0.77 \\
PS1-enriched & $<0.36$ & 0.33 & 0.61 \\
\hline
\end{tabular}

${ }^{a}$ Mean values from spectral addition and subtraction and from intersubtraction. ${ }^{b}$ Mean values for 12 -PCSL and 14-PCSL.

isomers of the phosphatidylcholine spin label agree rather well, indicating very little influence of the spin-label position on the lipid-protein interaction.

The mean values of the fraction of motionally restricted lipid for the different membrane systems are given in Table II. The mean values correspond to the average values obtained from the different subtraction methods and from spectral addition. For the phosphatidylcholine spin label, an average is also taken over the values for 12-PCSL and 14-PCSL, since these were not found to be appreciably different (cf. Table I).

The resolution of the fluid and motionally restricted lipid components indicates that the motions of the lipid chains are slowed down considerably in the thylakoid membranes by interaction with the integral membrane proteins. In principle, estimates of the relative rates of chain motion can be obtained from the line shapes of the individual spectral components. The spectra of the fluid lipid component indicate that certain components of the chain motion lie in the intermediate motional regime of nitroxide ESR spectroscopy. [For a complete analysis of spin-labeled chain motions in fluid lipid bilayers, see Lange et al. (1985)]. The spectra of the motionally restricted lipid component, on the other hand, show that essentially all components of the lipid chain motion lie close to the slow-motion regime of nitroxide ESR spectroscopy. This is especially clear in the case of the detergent-extracted PS2 subthylakoid fraction, which has a low lipid/protein ratio. The rates of at least some of the components of the lipid chain motion are therefore most appreciably slowed down by direct interaction with the intramembranous surface of the integral proteins.

\section{Discussion}

The results of the present study indicate that, in spite of the unusual lipid composition and specialized function of thylakoid membranes, lipid-protein interactions are manifest in a manner similar to that found previously for a wide range of biological membranes of nonplant origin [cf., e.g., Marsh (1983, 1985)]. Although one of the major thylakoid lipid components, namely, monogalactosyldiacylglycerol, is incapable alone of forming lamellar membrane structures, the basic features of the lipid-protein interactions remain the same in the lamellar membranes of intact thylakoids and subthylakoid membrane fragments as in other biomembranes. Similar results have been found in other membranes that contain nonlamellar forming phospholipids, for example, the rod outer segment disk mem-

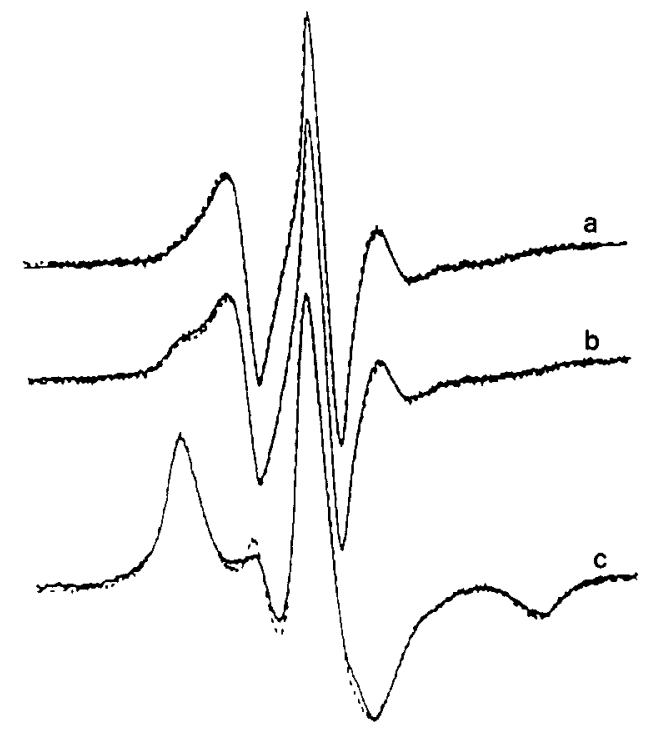

FIGURE 5: Spectral subtractions with the 12-MGDGSL monogalactosyl diacylglycerol spin label in thylakoid membranes at $18^{\circ} \mathrm{C}$ Full lines are either the experimental spectrum or single-component spectra used in the subtractions or addition. Dashed lines are either difference spectra or the addition spectrum. (a) Fluid component spectrum (full line) and difference spectrum (dashed line) obtained by subtracting $36 \%$ of the total intensity from spectrum $b$, using spectrum c. (b) Experimental membrane spectrum (full line) and composite spectrum (dashed line) obtained by adding $36 \%$ of the intensity of spectrum $\mathrm{c}$ and $64 \%$ of the intensity of spectrum a. (c) Motionally restricted component spectrum (full line) and difference spectrum (dashed line) obtained by subtracting $64 \%$ of the total intensity from spectrum $b$, using spectrum $a$. Total scan width was $100 \mathrm{G}$.

brane (Watts et al., 1979; Mollevanger \& De Grip, 1984), Na,K-ATPase membranes (Esmann et al., 1985; D. Marsh and M. Esmann, unpublished data), and cytochrome oxidase reconstituted with cardiolipin (Powell et al., 1985, and unpublished data).

The fraction of motionally restricted lipid, $f$, in the thylakoid membrane is expected to be related to the intramembranous surface area of the integral protein complexes. The mean molar ratio lipid/(protein/chlorophyll complex) in the thylakoid preparations is $346: 1$ when calculated from the data given under Materials and Methods with mean molecular weights of 0.9 and $623 \mathrm{kDa}$ for lipid and protein/chlorophyll complex, respectively (Murphy, 1986). The value of $f$ for the 12MGDGSL spin label is 0.36 , which would imply a mean number of motionally restricted lipids per protein of approximately 125 . The molecular topography of the thylakoid membrane has been reviewed by Murphy (1986). The average intramembranous diameter of the protein complexes is 110 $\AA$; thus, the mean number of lipids associated with the surface should be approximately 86 , assuming a mean diameter of 8.7 $\AA$ per lipid (which is determined by the lipid chains and therefore is independent of the headgroup). This value is considerably smaller than that deduced from the fraction of motionally restricted monogalactosyldiacylglycerol, even though the latter has the lowest selectivity of the spin-labeled lipids tested in the thylakoid membrane. Considerable uncertainty is associated with the theoretical estimate of the mean number of first-shell lipids around the protein, because of the complexity and heterogeneity of the system and the lack of detailed structural data. However, the results do suggest that monogalactosyldiacylglycerol does display some limited specificity for the protein component of the thylakoid membrane. The specificity for monogalactosyldiacylglycerol is the lowest of the lipids studied and comparable to that for phosphati- 
Table III: Selectivity of the Lipid-Protein Interactions in Thylakoid Membranes and Subthylakoid Membrane Fragments PS1 and PS2 ${ }^{a}$

\begin{tabular}{ccll}
\hline \multicolumn{1}{c}{ lipid } & MGDG & PC & PG \\
\hline thylakoids & & & \\
$n_{\mathrm{L}} / n_{\mathrm{t}}$ & 0.42 & 0.07 & 0.11 \\
$N_{\mathrm{L}} / n_{\mathrm{t}}$ & 0.15 & 0.03 & 0.06 \\
$K_{\mathrm{r}}$ & 1.7 & 1.9 & 3.4 \\
$K_{\mathrm{r}}(\mathrm{L}) / K_{\mathrm{r}}(\mathrm{PC})$ & 0.8 & 1.0 & 1.8 \\
$\mathrm{PS} 2-$ enriched & & & \\
$n_{\mathrm{L}} / n_{\mathrm{t}}$ & 0.46 & 0.04 & 0.10 \\
$N_{\mathrm{L}} / n_{\mathrm{t}}$ & 0.29 & 0.03 & 0.08 \\
$K_{\mathrm{r}}$ & 1.3 & 1.3 & 2.6 \\
$K_{\mathrm{r}}(\mathrm{L}) / K_{\mathrm{r}}(\mathrm{PC})$ & 1.0 & 1.0 & 2.0 \\
$\mathrm{PS} 1-$ enriched & & & \\
$n_{\mathrm{L}} / n_{\mathrm{t}}$ & 0.38 & 0.04 & 0.12 \\
$N_{\mathrm{L}} / n_{\mathrm{t}}$ & $<0.14$ & 0.01 & 0.07 \\
$K_{\mathrm{r}}$ & $<1.5$ & 1.3 & 4.2 \\
$K_{\mathrm{r}}(\mathrm{L}) / K_{\mathrm{r}}(\mathrm{PC})$ & $<1.1$ & 1.0 & 3.2 \\
\hline
\end{tabular}

${ }^{a} n_{\mathrm{L}} / n_{\mathrm{t}}$ is the fraction of lipid of type $\mathrm{L}$ in the total polar lipid of the thylakoid membrane (Chapman et al., 1983), and of the PS1-enriched or PS2-enriched membrane fragments (this work). $N_{\mathrm{L}} / n_{\mathrm{t}}\left(=f n_{\mathrm{L}} / n_{\mathrm{t}}\right)$ is an estimate of the fraction of the type $L$ that is motionally restricted, expressed relative to the total lipid. $K_{\mathrm{r}}$ is the relative association constant of the lipid of type L, calculated from eq 1 with $n_{\mathrm{t}}=346$ and $N_{1}$ $=86$ for thylakoid membranes, $n_{\mathrm{t}}=171$ and $N_{1}=74$ for the PS1-enriched membranes, and $n_{\mathrm{t}}=177$ and $N_{1}=100$ for the PS2-enriched membranes [cf. Murphy (1986)]. The ratio $K_{\mathrm{r}}(\mathrm{L}) / K_{\mathrm{r}}(\mathrm{PC})$ is independent of the choice of $n_{1}$ and $N_{1}$.

dylcholine, a lipid that is a major component of nonplant membranes in which it shows little specificity of interaction with the integral proteins [see, e.g., Marsh $(1983,1985)$ ]. Since monogalactosyldiacylglycerol is one the major lipid species in the thylakoid membrane, it is likely that it plays a structural role similar to that of phosphatidylcholine in mammalian membranes. Indeed, the propensity of monogalactosyldiacylglycerol to form nonlamellar phases suggests that this lipid may have a rather specific structural role in determining the geometry of the thylakoid membrane stacks.

The selectivity of the spin-labeled lipids can be quantitated in terms of the relative association constants derived from the equation for equilibrium exchange association with the protein (Brotherus et al., 1981; Marsh, 1985)

$$
K_{\mathrm{r}}=\left(n_{\mathrm{t}} / N_{\mathrm{l}}-1\right)\left(n_{\mathrm{b}}{ }^{*} / n_{\mathrm{f}}{ }^{*}\right)
$$

where $n_{\mathrm{t}}=345$ is the total mean lipid/protein ratio in the thylakoid membrane, $N_{1}=86$ is the mean number of first-shell lipid sites around the protein complex, and $n_{\mathrm{b}}{ }^{*} / n_{\mathrm{f}}{ }^{*}=f /(1-$ $f)$ is the ratio of the motionally restricted to fluid lipid components deduced from the ESR measurements. Values of the relative association constants for the different spin-labeled lipids in the thylakoid membrane, calculated in this way, are given in Table III. As expected from the preceding analysis, the relative association constant for monogalactosyldiacylglycerol is smallest, although greater than 1 , the value for phosphatidylcholine is slightly higher, and that for phosphatidylglycerol is approximately 3.5 relative to the mean background lipid. Corresponding values for the lipid selectivity in the PS1- and PS2-enriched membranes are also given in Table III. The lipid/(protein/chlorophyll) molar ratios, $n_{\mathrm{t}}$, again were calculated from the analytical data given under Materials and Methods and the mean molecular weights deduced by Murphy (1986). The values for the mean number of first-shell lipids, $N_{1}$, were also taken from this latter reference. The overall lipid specificity patterns are the same for the PS1- and PS2-enriched membrane fractions as for the whole thylakoid membrane. Monogalactosyldiacylglycerol and phosphatidylcholine display a smaller selectivity in the isolated membrane fractions than in the whole thylakoids: the value of the mean relative association constant is not much greater than that for the mean background lipid. The relative association constant for phosphatidylglycerol is the greatest of the lipids tested for both membrane fractions but is greater in the PS1-enriched fraction than in the PS2-enriched fraction, reflecting the different protein and/or lipid composition of the two photosystem complexes.

The lipid composition of the protein boundary layer shell can be estimated from the spin-label selectivity data together with the lipid composition of the native membrane (cf. Table III). The molar ratio MGDG/PC/PG for the total polar lipid in the thylakoid membrane is 6.0:1.0:1.6 (Chapman et al., 1983), whereas that at the lipid-protein interface is calculated to be 5.5:1.0:2.1 and that in the bulk lipid regions of the membrane is calculated to be $6 \cdot 3: 1.0: 1.2$. These different compositions reflect the mean selectivities of the different lipids for the various thylakoid protein complexes. Corresponding values for the total and boundary layer lipid compositions of the PS1- and PS2-enriched membranes are also given in Table III and were deduced from determinations made on the present preparations.

The relative lipid selectivity patterns in the different thylakoid membrane fractions also can be compared in terms of ratios of the relative association constants. The advantage of this method is that no assumptions have to be made regarding the effective protein size, mean molecular weights, etc., which were required in deriving the values of $K_{\mathrm{r}}$. For a given membrane system, the ratio of the relative association constants of the different lipids to that of a reference lipid, e.g., phosphatidylcholine, is given simply by the ratio of the corresponding values of $f /(1-f)$, as indicated by eq 1 . The values of $K_{\mathrm{r}}(\mathrm{L}) / K_{\mathrm{r}}(\mathrm{PC})$ obtained in this way are given for the different membranes also in Table III.

The finding of a specificity of interaction for phosphatidylglycerol in thylakoid membrane systems is particularly interesting. Although a selectivity for several different negatively charged lipids has been observed with most membrane protein systems studied to date (Marsh, 1987), the exception being rhodopsin, in none of these systems has a specificity been found for phosphatidylglycerol. This appears to be a special feature of the thylakoid membrane and, from the results of Table III, is seen to extend to the proteins of both the PS1 and PS2 reaction centers. As noted above, the relative association constant is greater in PS1-enriched membranes than in PS2-enriched membranes, although the phosphatidylglycerol (and sulfoquinovosyl diglyceride) content of the appressed and nonappressed regions of the thylakoid is rather similar (cf. Table III). This, therefore, indicates an intrinsically greater specificity of the proteins of the PS1-LHCl center for phosphatidylglycerol than of the PS2-LHC2 center proteins.

Phosphatidylglycerol has been found to be localized primarily to the outer monolayer of the thylakoid membrane [for references see Murphy (1986)]. It is possible that the protein selectivity for phosphatidylglycerol may in part be responsible for this transverse asymmetry, since the thylakoid proteins are vectorially incorporated into the lipid bilayer. A functional association of phosphatidylglycerol with the light-harvesting chlorophyll protein complexes of the thylakoid membrane has been suggested (Trëmolières et al., 1981; Rẻmy et al., 1982). The original finding was that this association specifically involves trans-hexadecenoic chains, but the association with phosphatidylglycerol headgroups also correlates well with the present spin-label results.

The different line shapes of the fluid and motionally restricted components indicate that the lipid chain mobility is substantially hindered by interaction with the hydrophobic 
surface of the protein. Previous spin-label studies by Hiller and Raison (1980), in which intact membranes were compared with dispersions of the extracted membrane lipids, are in qualitative agreement with this finding. By analogy with measurements of other lipid/protein systems (Marsh, 1983, 1985), it is not expected that the lipid chains are rigidly immobilized by interaction with the thylakoid proteins. The motionally restricted spectrum does display a temperature dependence, indicating significant motion on the conventional ESR time scale. The fact that exogeneously added spin label registers a motionally restricted component indicates that the motionally restricted lipids are free to exchange with the bulk membrane lipid environment. This finding is in agreement with previous measurements made by using radiolabeled lipids (Heinz \& Siefermann-Harms, 1981). Compared with the use of radiolabels, the spin-label method has the advantage of being able to differentiate the mobility states of the lipids. Measurements on the rate of exchange between the fluid and motionally restricted lipid components in other systems have yielded values in the region of $10^{7} \mathrm{~s}^{-1}$ for the intrinsic off-rate (Ryba et al., 1987; Horvăth et al., 1988a). It seems unlikely that these rates will be very appreciably lower in thylakoids, although they will of course reflect the selectivity for phosphatidylglycerol (Horváth et al., 1988b).

\section{ACKNOWLEDGMENTS}

Gang $\mathrm{Li}$ was supported by grants from the Education Ministry of China and the British Council.

\section{REFERENCES}

Arnon, D. I. (1949) Plant Physiol. 67, 1-15.

Berthold, D. A., Babcock, G. T., \& Yocum, C. F. (1981) FEBS Lett. 134, 231-234.

Brotherus, J. R., Griffith, O. H., Brotherus, M. O., Jost, P. C., Silvius, J. R., \& Hokin, L. E. (1981) Biochemistry 20, 5261-5267.

Chapman, D. J., De Felice, J., \& Barber, J. (1983) Plant Physiol. 72, 225-228.

Dunahay, T. G., Staehelin, L. A., Seiberg, M., Ogilvie, P. D., \& Berg, S. P. (1984) Biochim. Biophys. Acta 764, 179-193.

Esmann, M., Watts, A., \& Marsh, D. (1985) Biochemistry 24, 1386-1393.

Gounaris, K., \& Barber J. (1983) Trends Biochem. Sci. 8, 378-381.

Gounaris, K., Sundby, C., Andersson, B., \& Barber, J. (1983) FEBS Lett. 156, 170-174.

Gounaris, K., Barber, J., \& Harwood, J. L. (1986) Biochem. J. 237, 313-326.

Heinz, E., \& Siefermann-Harms, D. (1981) FEBS Lett. 124, $105-111$.
Hiller, R. G., \& Raison, J. K. (1980) Biochim. Biophys. Acta $599,63-72$.

Horväth, L. I., Brophy, P. J., \& Marsh, D. (1988a) Biochemistry 27, 46-52.

Horvāth, L. I., Brophy, P. J., \& Marsh, D. (1988b) Biochemistry 27, 5296-5304.

Knowles, P. F., Watts, A., \& Marsh, D. (1981) Biochemistry 20, 5888-5894.

Lange, A., Marsh, D., Wassmer, K.-H., Meier, P., \& Kothe, G. (1985) Biochemistry 24, 4383-4392.

Markwell, M. A. K., Haes, S. M., Tolbert, N. E., \& Bieber, L. L. (1981) Methods Enzymol. 72, 296-303.

Marsh, D. (1982) in Techniques in Lipid and Membrane Biochemistry (Hesketh, T. R., \& Metcalfe, J. C., Eds.) Vol. B4/II, pp B426/1-B426/44, Elsevier Ireland, Limerick.

Marsh, D. (1983) Trends Biochem. Sci. 8, 330-333.

Marsh, D. (1985) in Progress in Lipid-Protein Interactions (Watts, A., \& De Pont, J. J. H. H. M., Eds.) Vol. 1, pp 143-172, Elsevier, Amsterdam.

Marsh, D. (1987) J. Bioenerg. Biomembr. 19, 677-689.

Marsh, D., \& Watts, A. (1982) in Lipid-Protein Interactions (Jost, P. C., \& Griffith, O. H., Eds.) Vol. II, pp 53-126, Wiley-Interscience, New York.

Mollevanger, L. C. P. J., \& De Grip, W. J. (1984) FEBS Lett. 16, 256-260.

Murphy, D. J. (1986) Biochim. Biophys. Acta 864, 33-94.

Murphy, D. J., Cummins, I., \& Kang, A. S. (1989) Biochem. J. 258, 285-293.

Nishida, I., \& Yamada, M. (1985) Biochim. Biophys. Acta 813, 298-306.

Nishihara, M., Yokota, K., \& Kito, M. (1980) Biochim. Biophys. Acta 725, 104-112.

Powell, G. L., Knowles, P. F., \& Marsh, D. (1985) Biochim. Biophys. Acta 816, 191-194.

Rēmy, R., Trēmolières, A., Duval, J. C., Ambard-Bretteville, F., \& Dubacq, J.-P. (1982) FEBS Lett. 137, 271-275.

Ryba, N. J. P., Horvâth, L. I., Watts, A., \& Marsh, D. (1987) Biochemistry 26, 3234-3240.

Sane, P. V., Goodchild, D. J., \& Park, R. B. (1970) Biochim. Biophys. Acta 216, 162-178.

Telfer, A., Nicolson, J., \& Barber, J. (1976) FEBS Lett. 65, 77-83.

Trémolières, A., Dubacq, J.-P., Ambard-Bretteville, F., \& Rémy, R. (1981) FEBS Lett. 130, 27-31.

Waggoner, C., Aronson, H., More, J., \& Berg, S. P. (1985) Photosynth. Res. 6, 57-71.

Warden, J. T. (1978) Biol. Magn. Reson. 1, 239-275.

Watts, A., Volotovski, I. D., \& Marsh, D. (1979) Biochemistry $18,5006-5013$. 